INPLASY

PROTOCOL

To cite: Deng et al. Chinese herbal medicine for previous cesarean scar defect: $A$ protocol for systematic review and meta-analysis. Inplasy protocol 202090080. doi: 10.37766/inplasy2020.9.0080

Received: 22 September 2020

Published: 22 September 2020

Corresponding author:

Yong Jiang

jiangyong@pyztcm.com

Author Affiliation:

Chengdu University of

Traditional Chinese Medicine

Support: This work is supported by the National Natural Science Foundation of China (Grant No: 61801058).

Review Stage at time of this submission: Piloting of the study selection process.

Conflicts of interest:

\section{Chinese herbal medicine for previous cesarean scar defect: A protocol for systematic review and meta-analysis}

Deng, J1; Li, S2; Peng, Y3; Chen, Z4; Wang, C5; Fan, Z6; Zhao, M7; Jiang, $Y^{8} ;$ Wang, $Z^{9}$; Jiang, $Y^{10}$.

Review question / Objective: The study is aim to evaluate the efficacy and safety of the treatment that Chinese herbal medicine for PCSD.

Condition being studied: Previous cesarean scar defect (PCSD) is a gynecological disease that can causes bleeding after intercourse, prolonging menstrual period, intermenstrual bleeding, dysmenorrhea and other symptoms, and even lead to infertility. The treatment of this disease includes medicine and surgery, however, single western medicine treatment or surgical treatment has certain and clear shortcomings. In China and East Asia, Chinese medicine has been widely used to treat diverse diseases for thousands of years, and as an important means of treatment now, Chinese medicine plays a significant role in the treatment of gynecological diseases in China. The aim of this study is to assess the efficacy and safety of Chinese medicine for PCSD.

INPLASY registration number: This protocol was registered with the International Platform of Registered Systematic Review and Meta-Analysis Protocols (INPLASY) on 22 September 2020 and was last updated on 10 November 2020 (registration number INPLASY202090080).

There is no conflicts of interest

in this review.

\section{INTRODUCTION}

Review question / Objective: The study is aim to evaluate the efficacy and safety of the treatment that Chinese herbal medicine for PCSD.
Condition being studied: Previous cesarean scar defect (PCSD) is a gynecological disease that can causes bleeding after intercourse, prolonging menstrual period, intermenstrual bleeding, dysmenorrhea and other symptoms, and even lead to infertility. The treatment of this disease 
includes medicine and surgery, however, single western medicine treatment or surgical treatment has certain and clear shortcomings. In China and East Asia, Chinese medicine has been widely used to treat diverse diseases for thousands of years, and as an important means of treatment now, Chinese medicine plays a significant role in the treatment of gynecological diseases in China. The aim of this study is to assess the efficacy and safety of Chinese medicine for PCSD.

\section{METHODS}

Participant or population: Inclusion:1. History of cesarean section; 2. menostaxis $>7 d$; 3. PCSD diagnosed by B-ultrasound or hysteroscopy Exclusion: 1. Menostaxis caused by other reasons (like endocrine diseases, pregnancy, cancer, et al.) 2. Medicine allergy 3. Disobedience or lack of information.

Intervention: Chinese herbal medicine was the main intervention, like Chinese medicine versus Western medicine or Chinese medicine plus Western medicine versus placebo plus Western medicine.

Comparator: Placebo or other therapeutic agents.

Study designs to be included: Randomized controlled trials (RCTs) will be included.

Eligibility criteria: Types of studies. Randomized controlled trials (RCTs) in Chinese and English will be enrolled in this system review. Non-randomized controlled trials (non-RCTs), quasirandomized controlled trials (qRCTs), cohort studies, reviews, experimental studies, expert experience, case reports, the data of the included study is missing or incomplete, and duplicate publications will be excluded. Types of participants. All participants with PCSD will be included regardless of their nationality, occupation, educational background, belief, age, body or race. Patient and public involvement. This study has no patient and public involvement in consideration of this protocol for a systematic review. Types of interventions. All kinds of Chinese herbal medicine will be included, there are no restrictions on the amounts of herbs, methods of administration, dosage or duration of treatment. The comparsions will be either with other therapeutic agents or placebo.

Information sources: We will search the following databases for the indentification of RCTs: PubMed, the Cochrane Library, Chinese Biomedical Literature Database (CB), Chinese Science and Technique Journals Database (VIP), Excerpt Medica Database (EMBASE), Chinese National Knowledge Infrastructure Database (CNKI), and the Wanfang Database. All the above databases will be searched from the available date of inception until the latest issue (November 2020). We will search the reference lists of reviews and retrieve articles for additional studies on Google Scholar to identify further studies. We will include the literature published in journals and also "gray literature" such as degree theses and conference proceedings.

Main outcome(s): 1. The size of previous cesarean scar defect 2. Menstrual cycle 3. Menstrual phase 4. Menstrual volume 5. Duration of disease 6. Security index: general physical examination (temperature, pulse, repiration, blood, pressure), routine examination of blood, urine and stool, electrocardiogram, liver and kidney fuction examination.

Quality assessment / Risk of bias analysis: Two authors (ZC and ZW) will use the Cochrane tool of risk of bias to assess the risk of bias independently. The disagreement will be settled by another reviewer (YJ). We will evaluate the following contents: selection bias (random sequence generation, and allocation concealment), performance bias (blinding of participants and personnel), detection bias (blinding of outcome assessment), attrition bias (incomplete outcome data), reporting bias (selective outcome reporting), and other bias (other sources of bias). Studies will be evaluated high, low and unknown. 
Strategy of data synthesis: The metaanalysis in this review will use RevMan 5.3 software. Continuous variables will be reported as mean difference with $95 \%$ confidence intervals (Cls). For different measurement scales, we will use the standardized mean difference analysis with $95 \%$ Cls. Categorical variables will be summarized as risk ratios or odds ratio with $95 \% \mathrm{Cls}$. All analyses will be conducted in accordance with the Cochrane Handbook for Systematic Reviews of Interventions.

Subgroup analysis: If heterogeneity is detected, subgroup analysis will be performed to explore the differences in the methodologic quality, age, race/ethnicity, and types of Chinese medicine.

Sensibility analysis: Sensitivity analysis will be performed to examine the robustness of the result if there are sufficient studies included. The factors on effect are as follows: methodologic quality: analysis will be performed excluding studies of poor methodologic quality sample size: analysis will be performed excluding small sample size studies diagnostic criteria: analysis will be performed in studies of the same diagnostic criteria.

Country(ies) involved: China.

Keywords: Previous cesarean scar defect, Chinese herbal medicine, protocol, metaanalysis.

Contributions of each author:

Author 1 - Jiashuai Deng.

Author 2 - Sixuan Li.

Author 3 - Yangzhi Peng.

Author 4 - Zhaoxing Chen.

Author 5 - Changhong Wang.

Author 6 - Zhipeng Fan.

Author 7 - Mao Zhao.

Author 8 - Yuchang Jiang.

Author 9 - Zhaodi Wang.

Author 10 - Yong Jiang. 